\title{
Morphological and reproductive characterization of nascent allotetraploids cross-compatible with cultivated peanut (Arachis hypogaea L.)
}

\author{
Chandler Levinson (D) $\cdot$ Ye Chu $\cdot$ Xuelin Luo $\cdot$ H. Thomas Stalker $\cdot$ \\ Dongying Gao $\cdot$ C. Corley Holbrook $\cdot$ Peggy Ozias-Akins $(\mathbb{D}$
}

Received: 23 September 2020/Accepted: 1 March 2021/Published online: 21 March 2021

(C) The Author(s) 2021

\begin{abstract}
Peanut improvement is limited by a narrow genetic base. However, this obstacle can be circumvented by incorporating phenotypic variability from wild, diploid Arachis species through interspecific hybridizations. In this study, four allotetraploid interspecific hybrids $\operatorname{IpaCor}^{4 x}$ (A. ipaensis $\times$ A. correntina), $\operatorname{IpaDur}^{4 x}$ (A. ipaensis $\times$ A. duranensis), IpaSten $^{4 x}$ (A. ipaensis $\times A$. stenosperma), and ValSten $^{4 x}($ A. valida $\times$ A. stenosperma $)$ were created and morphologically characterized through the
\end{abstract}

C. Levinson · C. C. Holbrook · P. Ozias-Akins ( $\square)$ Institute of Plant Breeding, Genetics and Genomics, University of Georgia, Athens, GA, USA

e-mail: pozias@uga.edu

Y. Chu $\cdot$ P. Ozias-Akins

Horticulture Department, University of Georgia, Tifton, GA, USA

\section{Luo}

Experimental Statistics, University of Georgia, Tifton, GA, USA

\section{H. T. Stalker}

Crop and Soil Sciences, North Carolina State University, Raleigh, NC, USA

D. Gao

Center for Applied Genetic Technologies, University of Georgia, Athens, GA, USA

C. C. Holbrook

USDA - Agricultural Research Service, Crop Genetics and Breeding Research Unit, Tifton, GA, USA following parameters: flower count, flower size, flower banner pigmentation, leaf area and weight, leaf hairiness, main stem height, internode length, percent of reproductive nodes, biomass, 100 pod weight, and 100 seed weight. For every trait, except for flower banner absorption at $380 \mathrm{~nm}$, at least one or more allotetraploids differed from the cultivated peanut control. In general, these allotetraploids had a greater production of flowers during the growing season, larger flowers, larger and hairier leaves, taller main stems, longer primary laterals, longer internodes, lower percentage of reproductive nodes, heavier plant body masses, and smaller seeds and pods. This phenotypic diversity can be utilized directly in ornamental and forage breeding, while for oil and food crop breeding, this diversity will likely need to be selected against while desirable traits such as disease and insect resistance and abiotic stress tolerances derived from the wild diploid species are maintained.

Keywords Plant characterization · Phenotypic diversity $\cdot$ Introgression $\cdot$ Peanut breeding

\section{Introduction}

Peanut (Arachis hypogaea L.) is an allotetraploid species (AABB; $2 n=4 x=40$ ), and evidence suggests that it arose from a natural polyploidization of the 
diploid hybrid between A. ipaensis Krapov. and W.C. Gregory (BB; $2 n=2 x=20$ ) and A. duranensis Krapov. and W.C. Gregory (AA; $2 n=2 x=20$ ) (Bertioli et al. 2016). The difference in ploidy levels between cultivated peanut and its wild relatives resulted in a crossing barrier (Simpson 2001) and constricted genetic diversity in peanut. Subsequently, peanut cultivar improvement is limited by the narrow genetic base of this species (Holbrook et al. 2014). However, there are more than 80 wild Arachis species with a wide range of genotypic and phenotypic variability that can be used to expand peanut's genetic base (Stalker et al. 2016). The predominate use of Arachis species in peanut breeding programs has been for introgression of insect and disease resistances into cultivated peanut (Stalker et al. 2016); however, these materials could offer desirable phenotypic traits in addition to biotic and abiotic stress resistances and tolerances.

Due to peanut being an allotetraploid and most wild Arachis species being diploid, an efficient way to introgress genetic variability from wild Arachis species into cultivated peanut is to produce allotetraploid interspecific hybrids that are cross-compatible to peanut. This method has been used extensively in Brassica and is increasingly being used in peanut (Rahman 2013; Zhan et al. 2017; Leal-Bertioli et al. 2018; Ballén-Taborda et al. 2019). In addition to making the variability of the diploid Arachis species available for introgression into peanut, allotetraploids made from wild Arachis species can have additional phenotypic variability in plant morphology, physiology, anatomy and biochemistry, resulting from their new combination of genomes as well as from their increased ploidy level (Leal-Bertioli et al. 2017). For example, heterozygosity, subgenome interactions, and the gigas effect in allotetraploids have been shown to result in beneficial traits such as increased plant vigor and chlorophyll content, among other traits that peanut breeders could exploit in their breeding programs (Ramsey and Ramsey 2014; Leal-Bertioli et al. 2017).

Our peanut pre-breeding program seeks to produce neotetraploids from a selected set of diploid species as a genetic resource to enlarge the genetic variability of cultivated peanut. The selection of diploids was mainly based on their strong, diverse resistances to many insect and disease pests. Specifically, we chose A. correntina (Burkart) Krapov. and W.C. Gregory (PI 262808, GKP 9530), A. duranensis Krapov. and W.C.
Gregory (PI 468197, GKBSPSc 30060), A. ipaensis Krapov. and W.C. Gregory (PI 468322, GKBSPSc 30076), A. stenosperma Krapov. and W.C. Gregory (PI 666100, V10309), and A. valida Krapov. and W.C. Gregory (PI 468154, GK 30011), which have been reported to be resistant to aflatoxin, early leaf spot, late leaf spot, peanut rust, cylindrocladium black rot, groundnut rosette virus, tomato spotted wilt virus, peanut stunt virus, peanut mottle virus, nematodes, and numerous insect pests (Stalker 2017).

Efficient use of novel germplasm is dependent on the knowledge of the genetic and phenotypic diversity present in the materials. Germplasm characterization of agronomic traits is especially useful for germplasm intended to be used for crop improvement. However, there is a gap between the realized agronomic germplasm characterization and the needed level of characterization to identify and employ novel phenotypic variation in germplasm enhancement (Ferreira 2006). Therefore, when depositing the neotetraploids into a germplasm bank, it is important to provide a description of their morphological and reproductive characteristics and to inform breeders of the range of diversity presented in these materials. The goal of this study was to characterize morphological and reproductive traits of these nascent allotetraploids to promote efficient utilization of these materials in breeding programs after their release. This was done by building a catalog of descriptors, including flower count, flower size, flower banner pigmentation, leaf area and weight, leaf hairiness, main stem height, internode length, percentage of reproductive nodes, biomass, 100 pod weight, and 100 seed weight, of these materials.

\section{Materials and methods}

Plant materials

Three A genome diploid species, A. correntina (Burkart) Krapov. and W.C. Gregory (PI 262808, GKP 9530), A. duranensis Krapov. and W.C. Gregory (PI 468197, GKBSPSc 30060), and A. stenosperma Krapov. and W.C. Gregory (PI 666100, V10309), and one B genome species, A. ipaensis Krapov. and W.C. Gregory (PI 468322, GKBSPSc 300076) were crossed following the $\mathrm{BB} \times \mathrm{AA}$ crossing regime to create the diploid hybrids at North Carolina State University 
(Raleigh, NC). The A genome diploid species $A$. stenosperma Krapov. and W.C. Gregory (PI 666100, V10309) and the B genome diploid species A. valida Krapov. and W.C. Gregory (PI 468154, GK 30011) were crossed to create the diploid hybrids at the University of Georgia (Athens, GA). Four allotetraploids, IpaCor ${ }^{4 x}$, IpaDur ${ }^{4 x}$, IpaSten ${ }^{4 x}$, and ValSten $^{4 x}$, were created from the diploid hybrids by colchicine treatment at the University of Georgia Tifton Campus. The cultivated peanut check for these experiments was A. hypogaea '13-2113' [a selected runner-type breeding line from (C1805-617$2 \times$ 'Florida-07') $\times$ 'Georgia-06G']. C1805-617-2 is a selection from 'Tifguard' $\times$ 'Florida-07'.

$\mathrm{S}_{2}$ seeds from the four allotetraploids and seeds from 13-2113 were coated in Vitavax PC (Vitavax, Crompton, Middlebury, CT) and treated overnight in 0.5\% Florel Growth Regulator (Lawn and Garden Products Inc., Fresno, CA) to break dormancy on April 5th for the allotetraploids and April 26th, 2018, for 13-2113, respectively. The day after dormancy treatment, the seeds were planted in Jiffy pots filled with Promix growth medium (Premier Tech Horticulture, Quakertown, PA). On May 14th, the seedlings were transplanted to the center of the $1.82 \mathrm{~m}$ wide beds with $1 \mathrm{~m}$ distance between two neighboring plants and $4 \mathrm{~m}$ distance between plants in neighboring rows. The field was planted in a randomized complete block design with seven blocks, each containing one 13-2113, three IpaCor $^{4 x}$, three IpaDur ${ }^{4 x}$, one IpaSten ${ }^{4 x}$, and two ValSten $^{4 x}$ plants. Different numbers of plants per genotype were sown due to variation in seed availability. The field was treated with Provost fungicide spray four times each 2 weeks apart starting on June 12th. The allotetraploids grew large and started to intertwine against each other one month after transplanting. Since flower counts from individual plants would be inaccurate if the neighboring plants grew into each other, $30.8 \mathrm{~cm}$ tall Choppers garden edging (Emsco Group, Girard, PA) were installed to create a circle with a diameter of $1 \mathrm{~m}$ of edging around each plant to separate them. The garden hedges were installed in a circle with a radius of $0.5 \mathrm{~m}$ around each plant.
Characterization

\section{Flower count}

Numbers of flowers were counted from 50 to 108 days after transplanting between 8:30 and 11:00 am. Data collection was performed twice a week.

\section{Flower size}

Between 35 and 39 days after transplanting, four flowers were collected from each plant in $15 \mathrm{ml}$ falcon tubes (Corning CoStar, Corning, NY) containing a moist Kimwipe (Kimerly-Clark, Neenah, WI) to keep flowers from wilting during collection. Two flowers were taken from the primary lateral $(n+1)$ branch(es) and two from the secondary lateral $(n+2)$ branch(es). Flowers were dissected and scanned; measurements on hypanthium area $\left(\mathrm{cm}^{2}\right)$; banner area $\left(\mathrm{cm}^{2}\right)$; banner height $(\mathrm{cm})$, banner width $(\mathrm{cm})$, and left wing area $\left(\mathrm{cm}^{2}\right)$ were taken with ASSESS 2.0 software (APS Press).

\section{Flower banner pigmentation}

The flower banners dissected from the four flowers per plant previously used for flower size measurements were used to measure banner pigmentation. The banners were first weighed before they were put into $2 \mathrm{ml}$ tubes (Phenix Research, Swedesboro, NJ) with 4, $3.2 \mathrm{~mm}$ diameter, beads (BioSpec, Bartlesville, OK). The tubes were then submerged in liquid nitrogen and vortexed until the banner was a fine powder. The volume of $100 \%$ ethanol added to the pulverized banner was 10 times banner weight. The tubes where vortexed for about $3 \mathrm{~s}$ and then spun down at $3000 \mathrm{rpm}$ for $5 \mathrm{~min}$. $200 \mathrm{ul}$ of the supernatant was loaded into a spectrometry plate for reading, with $100 \%$ ethanol blanks as controls. Readings were normalized to the $100 \%$ ethanol, and only readings at wavelengths of $380,415,450,473$ and $495 \mathrm{~nm}$ were above the blank ethanol.

\section{Leaf area and weight}

At 101 and 102 days after transplanting, the first four newly emerged and fully expanded leaves on the main stem were collected from each plant and scanned for leaf area measurement using the ASSESS 2.0 software 
(APS Press). Leaf area was expressed as $\mathrm{cm}^{2}$. Fresh weights of the collected leaves were taken immediately after collection. The leaves were dried for $48 \mathrm{~h}$ at $30{ }^{\circ} \mathrm{C}$ and then dry weights were taken.

\section{Leaf hairs}

Between 114 and 119 days after transplanting, the most distal fully expanded leaflet was collected from two $n+1$ branches from each plant. The number of leaf hairs at the edge of the mature peanut leaflet within $1000 \mu \mathrm{m}$ was counted under the microscope.

\section{Main stem height}

Main stem height of plants was measured with a meter stick 91 days after transplanting when flowering in all genotypes plateaued.

\section{Internode length and differentiation of nodes}

The length of $\mathrm{n}+1$ branches was measured with meter sticks upon harvest, 120 days after transplanting. The total number of nodes on each branch was counted. The internode length was derived by dividing the branch length by the number of nodes.

The differentiation of reproductive and vegetative nodes was documented on the main stem and primary laterals. The presence or absence of flower buds and/or pegs was used to determine whether the node was reproductive or vegetative, respectively.

\section{Plant biomass}

After removing the pods, the above ground tissue of each plant was dried in the greenhouse $\left(25-32^{\circ} \mathrm{C}\right)$ for 7 days. Weight of dried tissue was taken.

\section{Pod and seed traits}

After harvest, pods were examined for the presence or absence of a beak, and 100 pod weight was measured. The pods were shelled, and 100 seed weight was taken. For lines that yielded less than 100 pods and/or seeds, 100 pod and seed weight were extrapolated by dividing the measured weight by the number of pods/seeds and multiplying by 100 . For $13-2113$, the seeds were bulked from the plants before 100 seed and 100 pod weight were taken; therefore, this genotype was excluded from the statistical analysis for these traits. However, the bulked 100 pod weight and 100 seed weight were plotted to give a reference point for the allotetraploids.

Statistical analysis

The flower count was analyzed using a standard twoway analysis of variance (ANOVA) with repeated measures. Genotype and days after transplanting were fixed effects, and block was a random effect. GLIMMIX procedure in SAS 9.4 (SAS Institute) was used to test the fixed effects. The residualnormality assumption was evaluated based on residual Q-Q plot, and the plot implied that the flower number was approximately normally distributed. Compound symmetry (CS) covariance structure was chosen to describe the correlation of flower counts between repeated measurements. One-way ANOVA was performed to determine the genotype effect on plant reproductive and morphological characteristics assessed using the following parameters: flower size, flower banner pigmentation, leaf area and weight, leaf hairiness, main stem height, internode length, percentage of reproductive nodes, biomass, 100 pod weight, and 100 seed weight. The IpaCor ${ }^{4 x}$, IpaDur ${ }^{4 x}$, and ValSten $^{4 x}$ replications in each block were averaged so that each allotetraploid had one data point per block and were equally represented in the statistical analysis. Means of each parameter among the treatments were separated based on the Tukey's Test $(\alpha=0.05)$ results.

\section{Results}

Flower count, size, and banner pigmentation

Genotype, days after transplanting, and genotype by days after transplanting interactions were all significant indicators of flower count (Table 1). IpaCor ${ }^{4 x}$ had the greatest flower counts from 50 to 108 days after transplanting (Fig. 1), with an average count of 47.45 flowers per day (Table 2). While IpaSten ${ }^{4 x}$ and ValSten $^{4 x}$ had fewer flowers than IpaCor ${ }^{4 x}$; their flower counts were stable throughout the data collection period with average flower counts of 14.54 and 35.57 per day, respectively (Table 2). IpaDur ${ }^{4 x}$ had a flowering trend similar to 13-2113, in which both had 
obvious decreasing counts beginning 78 days after transplanting, although this trend was even more prominent in 13-2113. IpaDur ${ }^{4 x}$ had an average flower count of 23.86 per day (Table 2). Overall, 13-2113 had the lowest average flower count at 11.50 flowers per day (Table 2).

Significant genotypic effects on hypanthium area, banner area, banner width, banner height, and leftwing area were found (Table 3). All the allotetraploids, except for IpaDur ${ }^{4 x}$, had significantly greater hypanthium area as compared to the cultivated control (Fig. 2a). IpaCor ${ }^{4 x}$ had the largest hypanthium area, in which the average area was more than three times that of the cultivated line. The trend in banner area and wing area were similar (Fig. 2b, c). IpaCor ${ }^{4 x}$ and ValSten $^{4 x}$ had significantly larger banner and wing area than the cultivated control, while IpaSten $^{4 x}$ was not statistically different from the cultivated control and $\operatorname{IpaDur}^{4 x}$ was even smaller than the cultivated control. All the genotypes had yellow wings, but only IpaSten $^{4 x}$ and ValSten ${ }^{4 x}$ had completely yellow banners, with thin red stripes originating from where the hypanthium and banner connect and spread outwards (Fig. 2d). 13-2113, IpaCor ${ }^{4 x}$, and IpaDur ${ }^{4 x}$ had banners with a yellow center and orange edge, though the intensity of the orange differed with IpaDur ${ }^{4 x}$ having a soft orange banner edge color, IpaCor ${ }^{4 x}$ a dark orange banner edge, and 13-2113 falling in between the soft orange of IpaDur ${ }^{4 x}$ and the dark orange of IpaCor ${ }^{4 x}$ (Fig. 2d).

Significant genotypic effects on banner pigment at the following wavelengths were found: $380,415,450$, 473, and $495 \mathrm{~nm}$ (Table 3). Although the banner pigment at 380 (ultraviolet-A) nm was above the $100 \%$ ethanol background, a significant genotypic effect on its absorbance was not detected. IpaSten ${ }^{4 x}$ and ValSten ${ }^{4 x}$, the two genotypes with yellow flowers (Fig. 2d), had the same level of absorbance at all the wavelengths (Fig. 3). At $415 \mathrm{~nm}$ (violet), the absorbance of all the genotypes was the same, except for IpaCor ${ }^{4 x}$, which had a lower absorbance than 13-2113, IpaDur ${ }^{4 x}$, and IpaSten ${ }^{4 x}$. The absorbance at both $450 \mathrm{~nm}$ (blue) and $473 \mathrm{~nm}$ (cyan) followed the same trend, in which only IpaCor ${ }^{4 x}$ and ValSten ${ }^{4 x}$ had significantly lower absorbance than 13-2113. Absorbance at $495 \mathrm{~nm}$ (cyan/green) was much lower for all the genotypes as compared to the other wavelengths, and only IpaSten ${ }^{4 x}$ and ValSten ${ }^{4 x}$ had significantly lower levels of absorbance than 13-2113.

Leaf area, weight, and hairiness

Significant genotypic effects on leaf area, fresh leaf weight, dry leaf weight, and leaf hairs were found (Table 3). All the allotetraploids had significantly larger and heavier leaves than the cultivated control as measured by leaf area and leaf weight (fresh and dry) (Fig. $4 \mathrm{a}-\mathrm{c}$ ). In addition, the range of leaf weight was much greater for all the allotetraploids as compared to the cultivated controls. All the allotetraploids had significantly greater numbers of hairs than 13-2113 (Fig. 4d, e).

\section{Plant size}

Significant genotypic effects on main stem height, main stem percentage of reproductive nodes, primary lateral length, internode length, primary lateral percentage of reproductive nodes, and plant biomass were found (Table 3). 13-2113 and IpaSten ${ }^{4 x}$ had the shortest main stem with an average height of 13.8 and $15.2 \mathrm{~cm}$, respectively (Fig. 5a). ValSten ${ }^{4 x}$ and IpaCor $^{4 x}$ had longer mainstems with an average of 22.0 and $27.1 \mathrm{~cm}$, respectively. However, IpaDur ${ }^{4 x}$ had the longest mainstem height by far with an average of $65.6 \mathrm{~cm}$, which was 4.75 times greater than 13-2113. Only IpaCor ${ }^{4 x}$ and IpaDur ${ }^{4 x}$ had reproductive nodes on their main stems, and IpaCor ${ }^{4 x}$ had the

Table 1 Type III tests of fixed effects of genotype, days after transplanting and genotype by days after transplanting interaction on flower count

\begin{tabular}{llll}
\hline Effect & $F$ or $X^{2}$ value & Df(n), df(d) & $P$ value \\
\hline Genotype & 21.17 & 4,23 & $<0.0001 * * *$ \\
Days after transplanting & 29.80 & 17,493 & $<0.0001 * * *$ \\
Genotype $\times$ days after transplanting & 7.98 & 68,493 & $<0.0001 * * *$ \\
\hline
\end{tabular}

$* \mathrm{P}<0.05, * * \mathrm{P}<0.01, * * * \mathrm{P}<0.001$ 
70

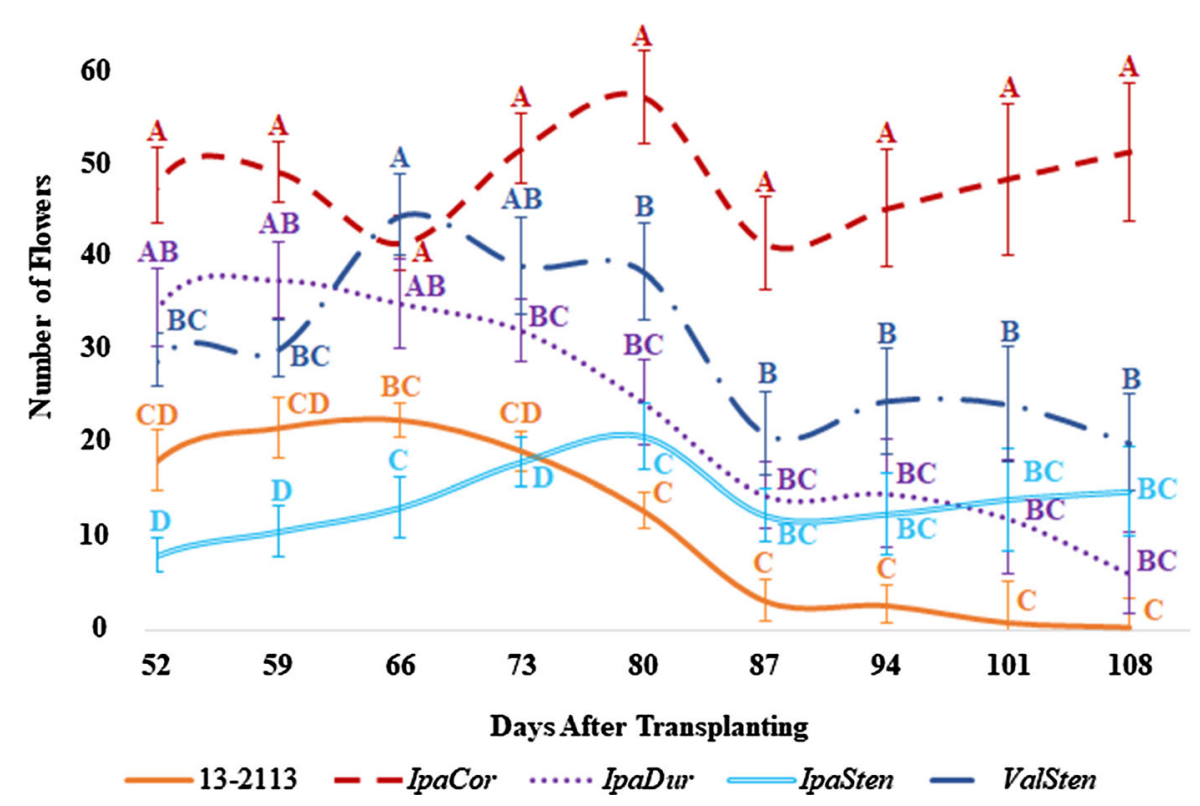

Fig. 1 Graph of number of flowers for each genotype over the course of 8 weeks, from 52 to 108 days after transplanting

Table 2 Tukey-Kramer grouping for genotype least squares means $($ alpha $=0.05)$ on flower count mean separation across all days after transplanting

\begin{tabular}{lll}
\hline Genotype & Estimated mean & Tukey-Kramer grouping \\
\hline $13-2113$ & 11.50 & $\mathrm{C}$ \\
IpaCor $^{4 x}$ & 47.45 & $\mathrm{~A}$ \\
IpaDur $^{4 x}$ & 23.87 & $\mathrm{BC}$ \\
IpaSten $^{4 x}$ & 14.54 & $\mathrm{C}$ \\
ValSten $^{4 x}$ & 30.59 & $\mathrm{~A}$ \\
\hline
\end{tabular}

highest percentage of reproductive nodes at $4.82 \%$ while IpaDur ${ }^{4 x}$ had a percentage of $0.87 \%$ (Fig. 5b). Primary lateral length did not follow the same trend as mainstem height, except that 13-2113 had the shortest primary lateral length at $41 \mathrm{~cm}$ (Fig. 5c). All the allotetraploids had much longer primary laterals than 13-2113. IpaSten ${ }^{4 x}$, with the shortest primary lateral length of the allotetraploids, still had a length 3 times longer than 13-2113. Meanwhile, ValSten ${ }^{4 x}$, with the longest primary lateral length of the allotetraploids, was 4.5 times longer than 13-2113. For internode length, 13-2113 was again classified as having the shortest of the five genotypes with an average internode length of $1.9 \mathrm{~cm}$ (Fig. 5d). All the allotetraploids had internode lengths at least 2 times greater than 13-2113. IpaDur ${ }^{4 x}$ and IpaSten $^{4 x}$ had greater internode lengths than IpaCor ${ }^{4 x}$, while ValSten $^{4 x}$ had the longest internode length overall. For primary lateral percentage of reproductive nodes, 13-2113 had the highest average at $43.2 \%$ (Fig. 5e). This percentage was about 3 times greater than the lowest percentage, which was IpaSten ${ }^{4 x}$ at $15.6 \%$, and about 1.5 times greater than the next highest percentage, which was ValSten $^{4 x}$ at $29.2 \%$. Lastly, 13-2113 and IpaSten ${ }^{4 x}$ had the lowest plant body mass at 90.3 and $144.7 \mathrm{~g}$, respectively (Fig. 5f). IpaDur ${ }^{4 x}$ and IpaCor $^{4 x}$ had plant biomass 2 times greater than 13-2113, while ValSten $^{4 x}$ was 3 times greater than 13-2113.

\section{Pod and seed weight}

Significant genotypic effects on allotetraploid 100 pod and seed weights were not found (Table 3). All the allotetraploids had low 100 pod weights ranging from the lowest at $21.2 \mathrm{~g}$ for IpaCor ${ }^{4 x}$ to the highest at 25.4 $\mathrm{g}$ for ValSten $^{4 x}$ (Fig. 6a, c). These weights were only $24-29 \%$ of the weight $(86.9 \mathrm{~g}$ ) for 13-2113. Similarly, all the allotetraploids had low 100 seed weight ranging from the lowest at $14.4 \mathrm{~g}$ for IpaSten ${ }^{4 x}$ to the highest at 
Table 3 ANOVA output testing the genotype effects on plant reproductive and morphological

characteristics assessed using the following parameters: flower size, flower banner pigmentation, leaf area and weight, leaf hairiness, main stem height, internode length, percentage of reproductive nodes, biomass, 100 pod weight, and 100 seed weight
$* P<0.05, * * P<0.01$,

$* * * P<0.001$

${ }^{\mathrm{a}} 13-2113$ was not included in 100 seed and 100 pod analysis

\begin{tabular}{|c|c|c|c|}
\hline Parameter & $F$ or $X^{2}$ value & $\operatorname{Df}(\mathrm{n}), \mathrm{df}(\mathrm{d})$ & $P$ value \\
\hline Hypanthium area & 57.11 & 4,28 & $<0.0001 * * *$ \\
\hline Banner area & 47.15 & 4,28 & $<0.0001 * * *$ \\
\hline Banner width & 55.53 & 4,28 & $<0.0001 * * *$ \\
\hline Banner height & 43.77 & 4,28 & $<0.0001 * * *$ \\
\hline Left wing area & 49.85 & 4,28 & $<0.0001 * * *$ \\
\hline Banner pigmentation: $380 \mathrm{~nm}$ & 2.40 & 4,29 & 0.073 \\
\hline Banner pigmentation: $415 \mathrm{~nm}$ & 5.15 & 4,29 & $0.0029 * *$ \\
\hline Banner pigmentation: $450 \mathrm{~nm}$ & 4.19 & 4,29 & $0.0035 * *$ \\
\hline Banner pigmentation: $473 \mathrm{~nm}$ & 4.19 & 4,29 & $0.0085 * *$ \\
\hline Banner pigmentation: $495 \mathrm{~nm}$ & 16.11 & 4,29 & $<0.0001 * * *$ \\
\hline Leaf area & 10.12 & 4,25 & $<0.0001 * * *$ \\
\hline Leaf weight (fresh) & 12.73 & 4,25 & $<0.0001 * * *$ \\
\hline Leaf weight (dry) & 8.34 & 4,25 & $0.0003 * * *$ \\
\hline Leaf hair & 9.47 & 4,29 & $<0.0001 * * *$ \\
\hline Main stem height & 211.33 & 4,25 & $<0.0001 * * *$ \\
\hline Primary lateral length & 26.71 & 4,29 & $<0.0001 * * *$ \\
\hline Internode length & 35.25 & 4,29 & $<0.0001 * * *$ \\
\hline Percent reproductive nodes (n) & 4.16 & 4,29 & $0.0088 * *$ \\
\hline Percent reproductive nodes $(n+1)$ & 21.39 & 4,29 & $<0.0001 * * *$ \\
\hline Plant biomass & 9.27 & 4,29 & $<0.0001 * * *$ \\
\hline 100 pod weight & 1.45 & $3,20^{\mathrm{a}}$ & $0.26^{\mathrm{a}}$ \\
\hline 100 seed weight & 0.55 & $3,21^{\mathrm{a}}$ & $0.65^{\mathrm{a}}$ \\
\hline
\end{tabular}

$16.5 \mathrm{~g}$ for ValSten $^{4 x}$ (Fig. 6b, c). These weights were only $19-22 \%$ of the weight (76.6g) for 13-2113.

\section{Discussion}

Wild Arachis species have a wide range of genetic and phenotypic variability, including strong resistances to many economically devastating insect and disease pests (Stalker 2017). This variability presents an opportunity to both widen genetic diversity in peanut and to introgress phenotypic traits such as increased plant biomass, flower count, and disease and insect resistances into cultivated peanut. Some species such as $A$. cardenasii have already been used successfully to introgress leaf spot and nematode resistance into cultivated peanut, demonstrating the value of these species for introgression of desirable traits (Simpson et al. 2003; Tallury et al. 2014). The four allotetraploids reported in the study are cross compatible to cultivated peanut and therefore, they can be readily used for peanut cultivar improvement. The documentation of the morphological and reproductive characterization of these materials allows phenotypic traits such as plant vigor (demonstrated by increased plant biomass, plant height, flower production, among others) to be introgressed into peanut breeding lines. In the case of undesirable phenotypic traits characterized for these materials, plans for selection against these traits should be considered.

To fill the gap in characterization of these neotetraploids, they were examined in-depth for both morphological and reproductive characteristics. For most of the traits, at least one or more allotetraploid differed from the cultivated peanut control; therefore, these materials offer a wide range of phenotypic diversity that can be utilized for peanut breeding. Overall, the allotetraploids tended to have more flowers over the growing season, larger flowers, larger and hairier leaves, taller main stems, longer primary laterals, longer internodes, lower percentages of reproductive nodes, heavier plant body masses, and smaller seeds and pods. Greater leaf hair density may be a trait that breeders choose to select for in order to increase resistance to some insect pests, since hair density has been demonstrated as one defense 

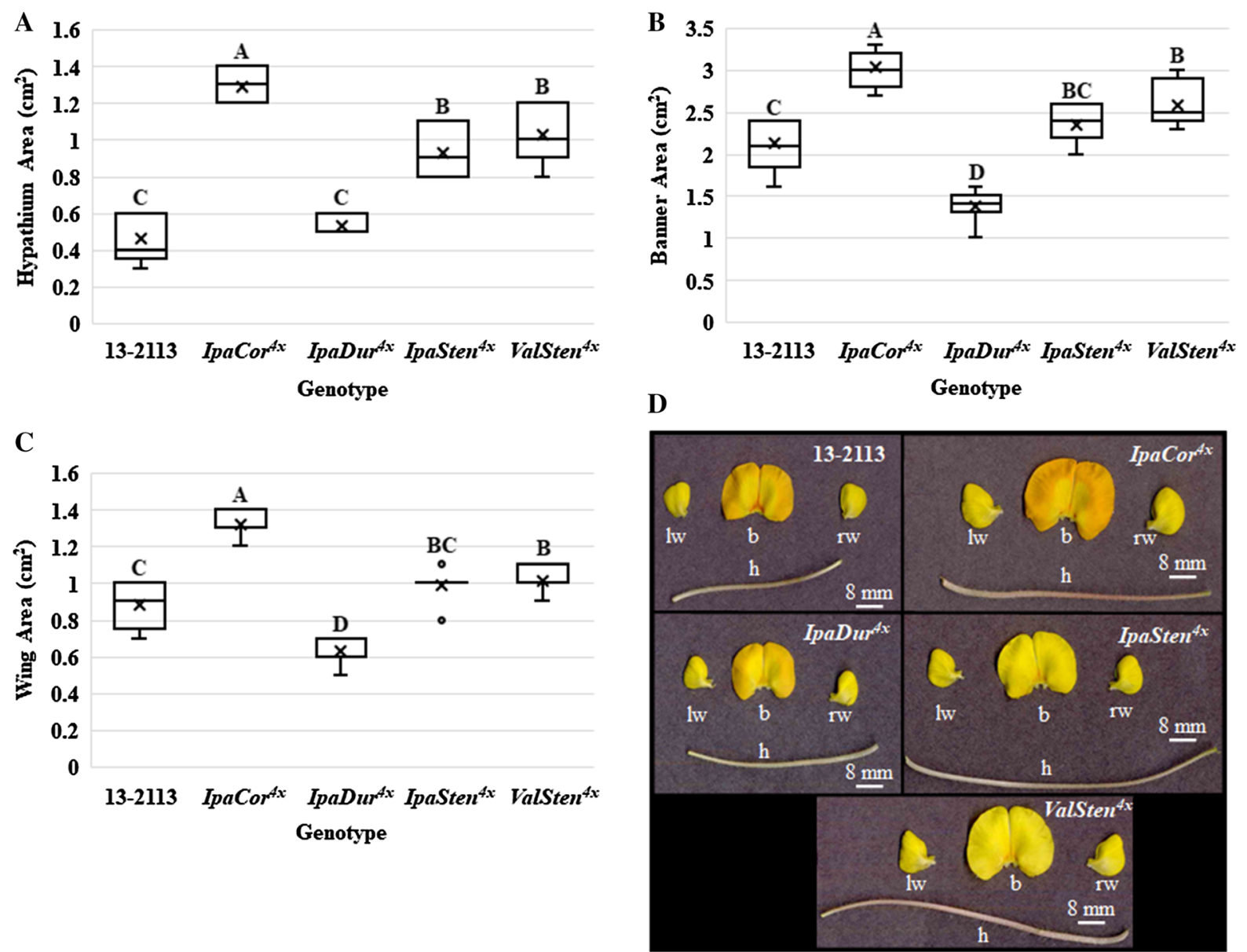

Fig. 2 Box plots of a hypanthium area, $\mathbf{b}$ banner area, and $\mathbf{c}$ wing area $\mathbf{d}$ Scans of dissected flowers from secondary lateral branches, in which banner is labeled b, hypanthium $\mathrm{h}$, left wing lw, and right-wing rw

mechanism to some insect pests (Mohammad et al. 2019). In addition, the allotetraploids with larger flowers and more flowers over the growing season such as IpaCor ${ }^{4 x}$ IpaSten $^{4 x}$, and ValSten ${ }^{4 x}$ could be used for ornamental breeding. This absence of decline in flower production demonstrated by these three allotetraploids is likely influenced by the perenniality of A. correntina and A. stenosperma (Stalker and Simpson 1995). Likewise, allotetraploids with heavy plant biomass such as IpaCor ${ }^{4 x}$, IpaDur ${ }^{4 x}$, and ValSten $^{4 x}$ could be used for forage breeding. On the contrary, breeders aiming to introgress biotic and abiotic stress resistances and tolerances into cultivars used as oil or food crop will need to backcross these materials to their desired peanut cultivars. Linkage drag for small pod and seed size, low percentage of reproductive nodes, long primary lateral length, and long internode length should be selected against in each breeding cycle.

The findings of this study complement a previous report Leal-Bertioli et al. (2017), which compared 26 anatomical, morphological, and physiological traits of six Arachis neotetraploids, their parental diploids, and A. hypogaea grown in a greenhouse environment. Despite different growing environments (greenhouse versus the field) and testing different allotetraploids, both Leal-Bertioli et al. (2017) and this study found that allotetraploids had high levels of plant vigor and biomass. Leal-Bertioli et al. (2017) reported this greater vigor by increased stem height, aerial dry biomass, leaf size, leaf canopy, root biomass, and leaf cell size as compared to the parental diploids, while this study identified increased vigor by increased flower production through the growing season, flower 

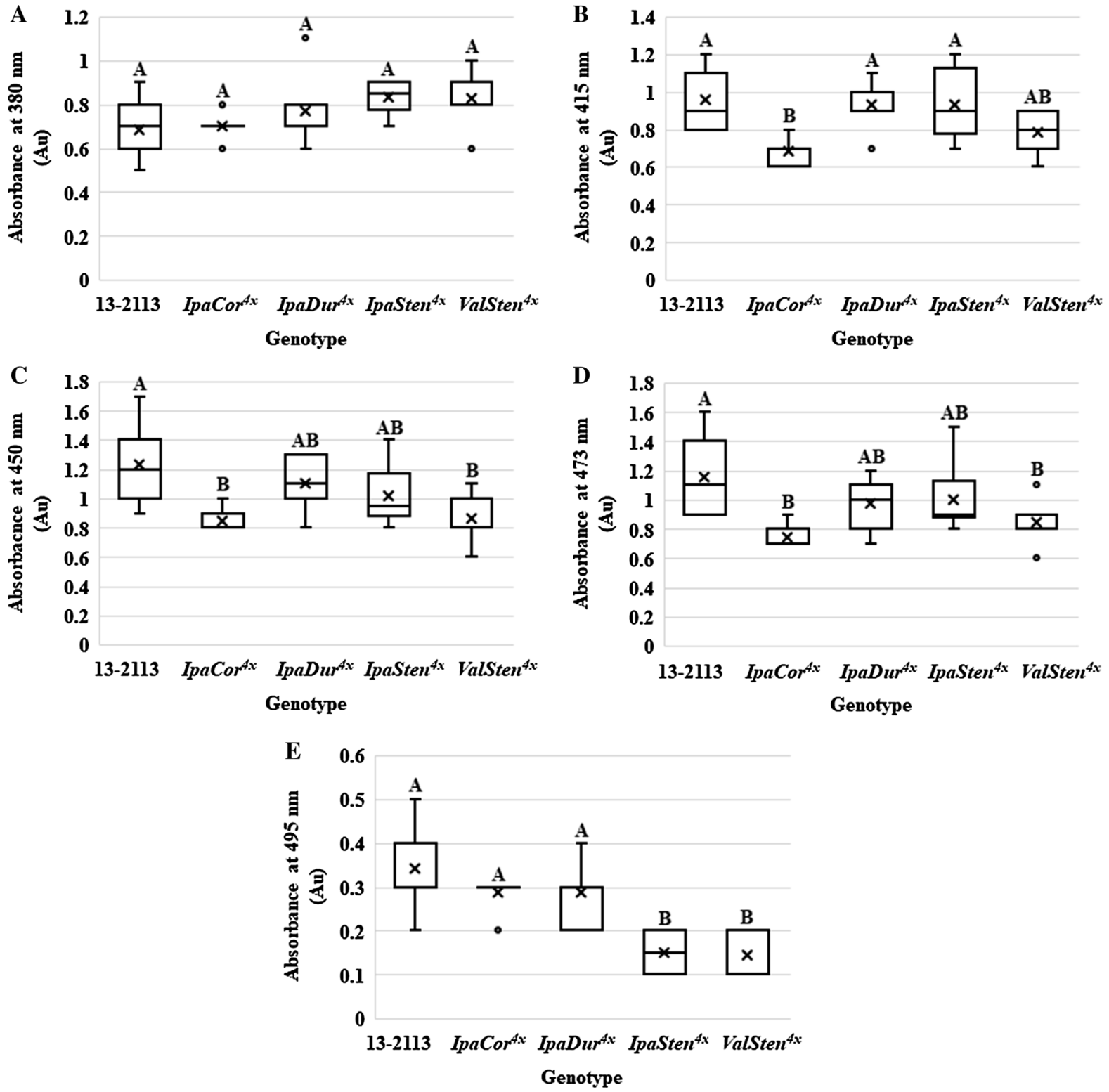

Fig. 3 Box plots of absorbance at a 380, b 415, c 450, d 473, and e $495 \mathrm{~nm}$

size, leaf area and weight, main stem height, internode length, and plant biomass as compared to a peanut breeding line. In addition, both Leal-Bertioli et al. (2017) and this study found that seed size and yield of these allotetraploids were small. The increased plant vigor of allotetraploids does not seem to be correlated to reproductive vigor; therefore, plant breeders will have to backcross these materials to peanut breeding lines while selecting against small seed size, low yield, and fragile pegs.
More recent studies have begun to characterize biotic and abiotic stress resistance and tolerance levels of Arachis-derived allotetraploids to inform breeders of the value of these materials for traits such as late leaf spot and rust resistance, nematode resistance, and drought stress tolerance (Dutra et al. 2018; LealBertioli et al. 2018). Leal-Bertioli et al. (2018) and Ballén-Taborda et al. (2019) built upon the previously discussed report Leal-Bertioli et al. (2017) and found that the IpaDur $^{4 x}$ V14167 allotetraploid had wild 

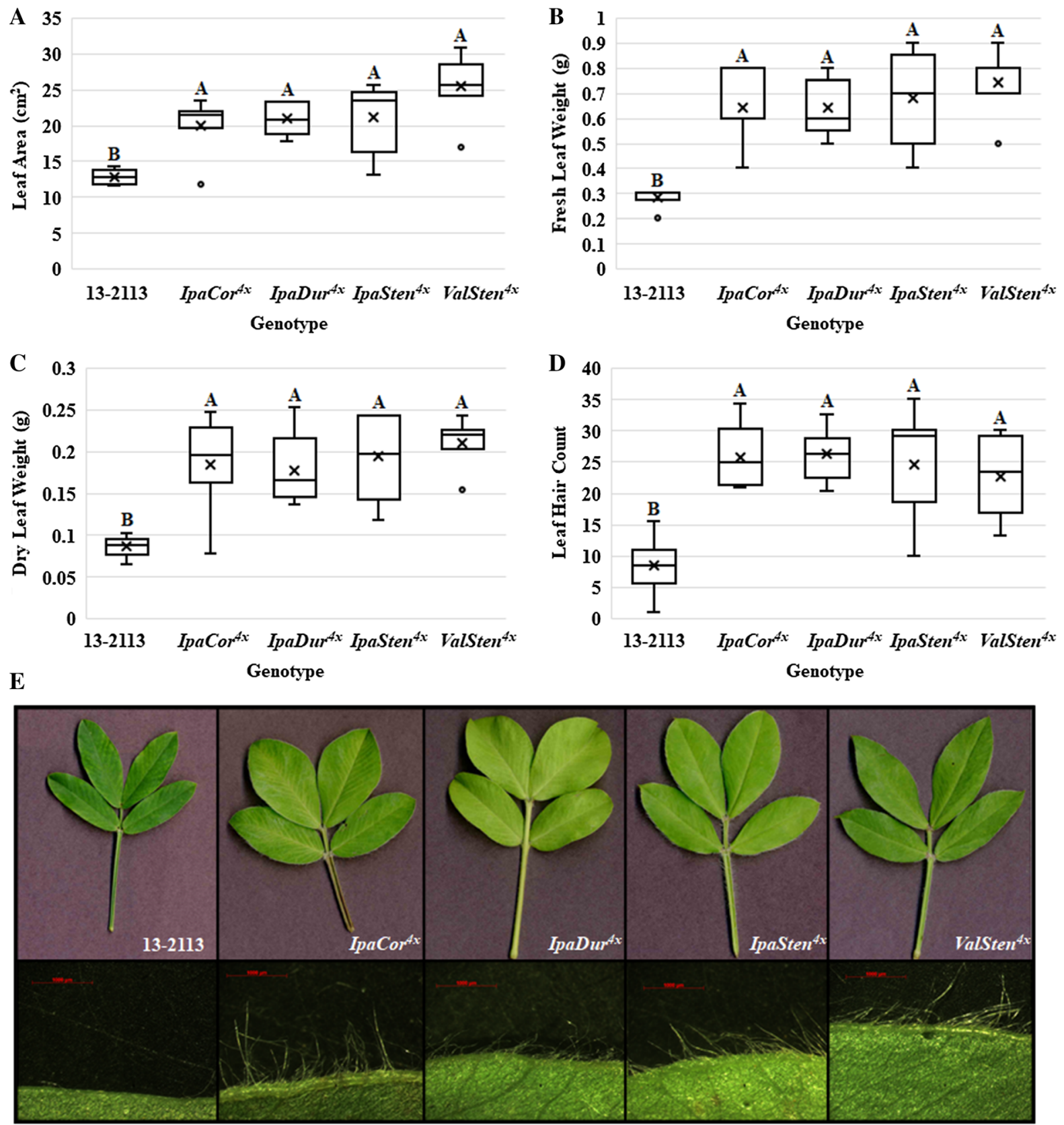

Fig. 4 Box plots of a leaf area, $\mathbf{b}$ fresh leaf weight, $\mathbf{c}$ dry leaf weight, and $\mathbf{d}$ leaf hair count $\mathbf{e}$ Scans of the most distal, fully expanded leaf excised from the mainstem (top) and pictures of the edge of the bottom, right leaflet with a 1000 um scale bar in red

Arachis species traits such as long branches and small seeds but higher levels of late leaf spot and rust resistance as compared to Runner-886, a susceptible control. The IpaDur ${ }^{4 x} 30060$ in this study was produced from a cross between A. ipaensis and a different accession of $A$. duranensis than the accession Leal-Bertioli et al. (2018) used. This is an important distinction, since resistance to pests is variable between accessions within an Arachis species (Stalker and Campbell 1983). One example of variation for resistance within a species is A. correntina. Stalker and Campbell (1983) identified A. correntina 9530 (PI 262808), the same accession used in this study to make the $\operatorname{IpaCor}^{4 x}$ allotetraploid, as well as A. correntina 

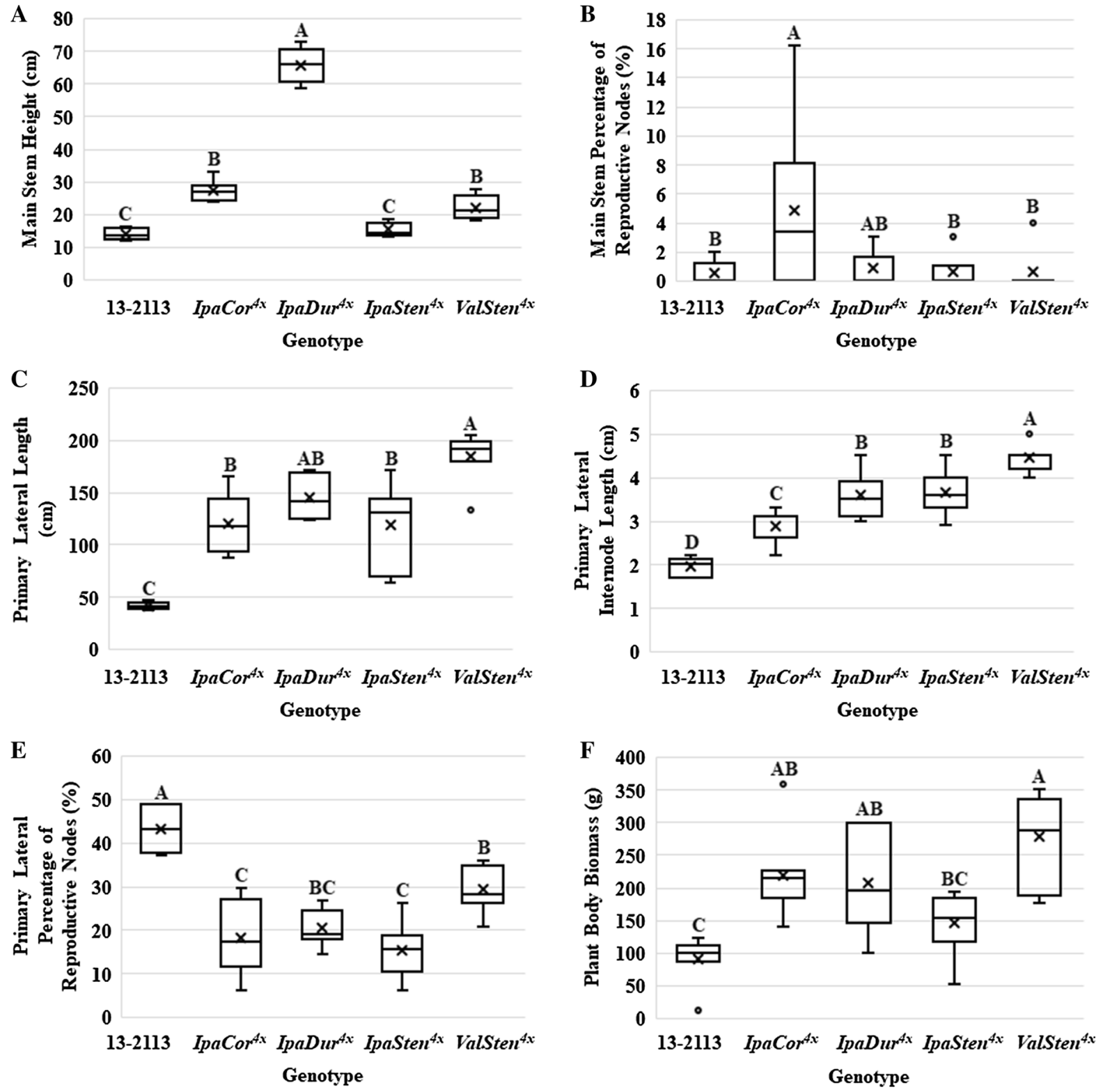

Fig. 5 Box plots of a mainstem height, $\mathbf{b}$ main stem percentage of reproductive node, $\mathbf{c}$ primary lateral length, $\mathbf{d}$ primary lateral internode length, e primary lateral percentage of reproductive nodes, and $\mathbf{f}$ plant body biomass

(Manfredi \#5 and \#36) as sources for potato leafhopper resistance, but only A. correntina 9530 and Manfredi \#36 as sources for corn earworm resistance. Furthermore, another study identified A. correntina 9548 (PI 262881) as a source of resistance to two spotted spider mites but not Manfredi \#36 (Johnson et al. 1977). Therefore, producing allotetraploids with the same species but different accessions creates an even greater resource pool for breeders and is not redundant.

The genetic resources produced in this study widen the available pool of diversity for peanut breeders for a wide range of phenotypic traits. The future direction of this research is to continue the momentum of using this allotetraploid method to bridge wild diploid species with tetraploid peanut to reach the success that has been achieved in Brassica. Therefore, these materials 

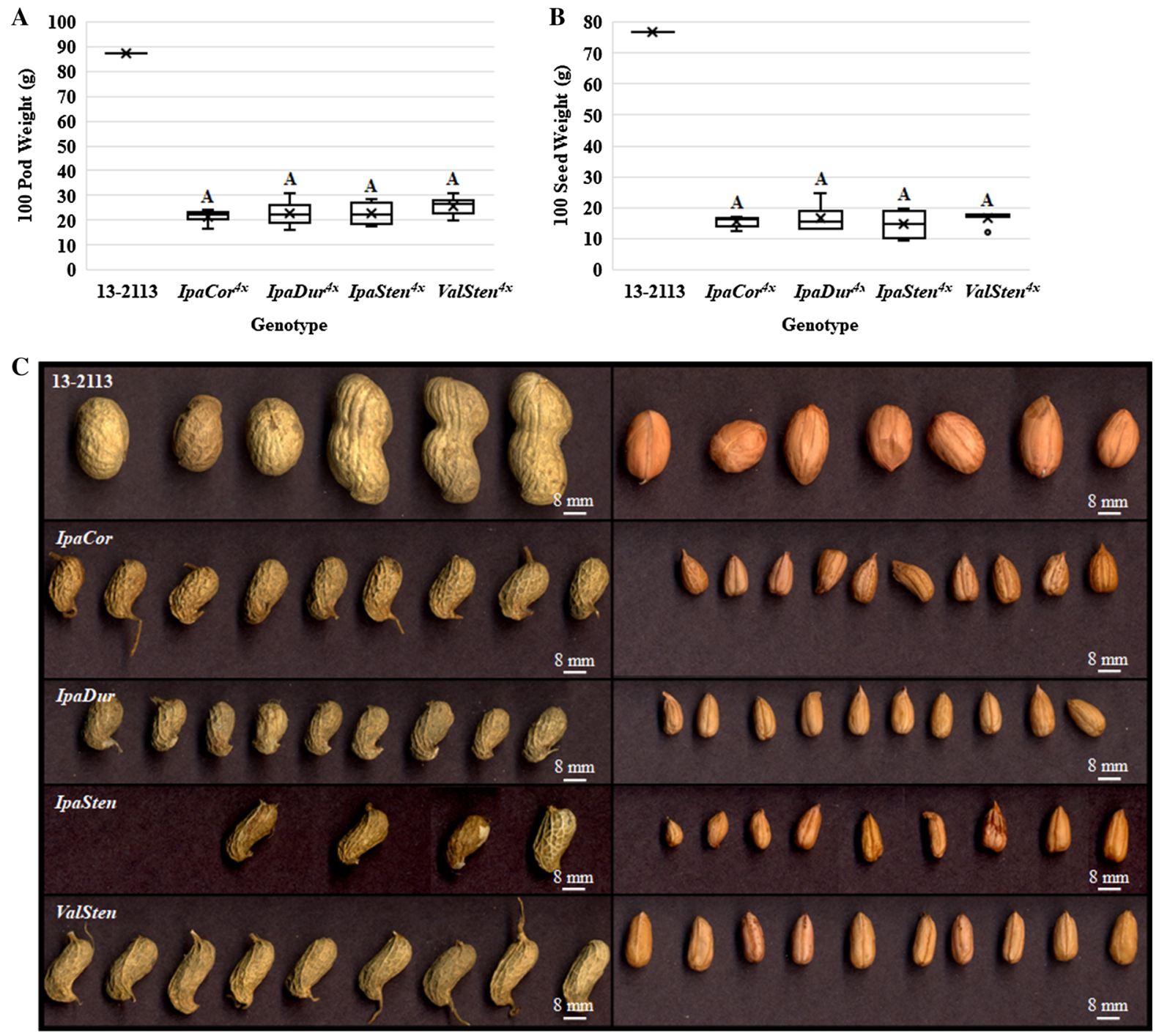

Fig. 6 Box plots of a 100 pod weight and b 100 seed weight $\mathbf{c}$ scans of pods (left) and seeds (right)

and data are being provided to a germplasm bank to allow breeders the opportunity to use and mine these materials for their phenotypic diversity. In addition, we will study the disease and insect resistance levels in these materials so that breeders can use these materials for resistance introgression in addition to introgression of morphological and reproductive traits. Populations of these allotetraploids crossed to peanut breeding lines could be produced so that QTL of these morphological and reproductive traits can be mapped and shared with breeders to make introgression more efficient.

\section{Conclusions}

Comprehensive morphological and reproductive characterization of four unique allotetraploids was performed from field grown materials in this study. Most of these allotetraploids produced more flowers over the growing season, larger flowers, larger and hairier leaves, taller main stems, longer primary laterals, longer internodes, lower percentages of reproductive nodes, heavier plant body masses, and smaller seeds and pods than cultivated peanut, although there were exceptions for certain allotetraploids. These germplasm resources will be released so that further 
characterization and utility in peanut breeding will become possible to improve peanut production.

Acknowledgements This work was supported by the National Science Foundation (Grant \# MCB-1543922) and by the AFRI NIFA Fellowships Grant Program: Predoctoral Fellowships project Accession No. 1019105 from the USDA National Institute of Food and Agriculture. The authors would like to express their appreciation to Micah Levinson of the Department of Horticulture, University of Georgia, for assistance in data collection.

Author contributions CL, YC, and PO-A contributed to the study conception and design. Creation of the materials was performed by TS, YC, CL, DG, and CH. Data collection was performed by Chandler Levinson. Data analysis was performed by YC, XL, and CL. The manuscript was written by CL, and all authors commented on previous versions of the manuscript. All authors read and approved the final manuscript.

Funding This work was supported by the National Science Foundation (Grant \# MCB-1543922) and by the AFRI NIFA Fellowships Grant Program: Predoctoral Fellowships project accession no. 1019105 from the USDA National Institute of Food and Agriculture.

Availability of the data and material The datasets generated and analyzed during the current study are available from the corresponding author on reasonable request. Seeds of IpaCor (PI 695391), IpaDur (PI695392), and ValSten (PI 695393) have been deposited with the USDA Plant Genetic Resources and Conservation Unit (Griffin, GA) and the National Laboratory for Genetic Resources Preservation (Fort Collins, CO).

\section{Declarations}

Conflicts of interest The authors report no conflicts of interest.

Open Access This article is licensed under a Creative Commons Attribution 4.0 International License, which permits use, sharing, adaptation, distribution and reproduction in any medium or format, as long as you give appropriate credit to the original author(s) and the source, provide a link to the Creative Commons licence, and indicate if changes were made. The images or other third party material in this article are included in the article's Creative Commons licence, unless indicated otherwise in a credit line to the material. If material is not included in the article's Creative Commons licence and your intended use is not permitted by statutory regulation or exceeds the permitted use, you will need to obtain permission directly from the copyright holder. To view a copy of this licence, visit http://creativecommons.org/licenses/by/4.0/.

\section{References}

Ballén-Taborda C, Chu Y, Ozias-Akins P, Timper P, Holbrook CC, Jackson SA, Bertioli DJ, Leal-Bertioli SCM (2019) A new source of root-knot nematode resistance from Arachis stenosperma incorporated into allotetraploid peanut (Arachis hypogaea). Sci Rep 9:17702. https://doi.org/10.1038/ s41598-019-54183-1

Bertioli DJ, Cannon SB, Froenicke L, Huang G, Farmer AD, Cannon EK, Liu X, Gao D, Clevenger J, Dash S, Ren L, Moretzsohn MC, Shirasawa K, Huang W, Vidigal B, Abernathy B, Chu Y, Niederhuth CE, Umale P, Araujo AC, Kozik A, Do Kim K, Burow MD, Varshney RK, Wang X, Zhang X, Barkley N, Guimaraes PM, Isobe S, Guo B, Liao B, Stalker HT, Schmitz RJ, Scheffler BE, Leal-Bertioli SC, Xun X, Jackson SA, Michelmore R, Ozias-Akins P (2016) The genome sequences of Arachis duranensis and Arachis ipaensis, the diploid ancestors of cultivated peanut. Nat Genet 48:438-446. https://doi.org/10.1038/ng.3517

Dutra WF, Yrlânia LG, Ramos JPC, Fernandes PD, Silva CRC, Bertioli DJ, Leal-Bertioli SCM, Santos RC (2018) Introgression of wild alleles into the tetraploid peanut crop to improve water use efficiency, earliness and yield. PLoS ONE 13(6):e0198776. https://doi.org/10.1371/journal. pone.0198776

Ferreira ME (2006) Molecular analysis of gene banks for sustainable conservation and increased use of crop genetic resources. In: Ruane J, Sonnino A (eds) The role of biotechnology in exploring and protecting agricultural genetic resources. Food and Agriculture Organization of the United Nations, Rome, pp 121-128

Holbrook CC, Brenneman TB, Stalker HT, Johnson WC, OziasAkins P, Chu Y, Vellidis G, McClusky D (2014) Peanut. In: Smith S, Diers B, Specht J, Carver B (eds) Yield gains in major U.S. field crops. ASA-CSSA-SSSA, Madison, pp 173-194

Johnson DR, Wynne JC, Campbell WV (1977) Resistance of wild species of Arachis to the twospotted spider mite, Tetranychus urticae. Peanut Sci 4:9-11

Leal-Bertioli SCM, Moretzsohn MC, Santos SP, Brasileiro ACM, Guimarães PM, Bertioli DJ, Araujo ACG (2017) Phenotypic effects of allotetraploidization of wild Arachis and their implications for peanut domestication. Am J Bot 104(3):379-388

Leal-Bertioli SCM, Godoy IJ, Santos JF, Doyle JJ, Guimarães PM, Abernathy BL, Jackson SA, Moretzsohn MC, Bertioli DJ (2018) Segmental allopolyploidy in action: Increasing diversity through polyploid hybridization and homoeologous recombination. Am J Bot 105(6):1-14. https://doi. org/10.1002/(ISSN)1537-2197

Mohammad SA, Gopalakrishna NK, Tippannavar PS, Nadaf HL (2019) Biophysical and biochemical mechanism of resistance to Spodoptera litura in groundnut (Arachis hypogaea L.). J Entomol 7(4):86-96

Rahman H (2013) Review: Breeding spring canola (Brassica napus L.) by the use of exotic germplasm. Can J Plant Sci 93(3):363-373. https://doi.org/10.4141/cjps2012-074

Ramsey J, Ramsey TS (2014) Ecological studies of polyploidy in the 100 years following its discovery. Philos Trans R Soc 369:20130352. https://doi.org/10.1098/rstb.2013.0352 
Simpson CE (2001) Use of wild Arachis species/introgression of genes into A. hypogaea L. Peanut Sci 26:114-116. https:// doi.org/10.3146/i0095-3679-28-2-12

Simpson CE, Starr JL, Church GT, Burow MD, Paterson AH (2003) Registration of 'Nema TAM' peanut. Crop Sci 33:1418

Stalker HT (2017) Utilizing wild species for peanut improvement. Crop Sci 57:1102-1120. https://doi.org/10.2135/ cropsci2016.09.0824

Stalker HT, Campbell WV (1983) Resistance of wild species of peanut to an insect complex. Peanut Sci 10(1):30-33

Stalker HT, Simpson CE (1995) Genetic resources in Arachis. In: Pattee HE, Stalker HT (eds) Advances in peanut science. Am Peanut Res Educ Soc, Stillwater, pp 14-53

Stalker HT, Tallury SP, Seijo GR, Leal-Bertioli SC (2016) Biology, speciation, and utilization of peanut species. In: Stalker HT, Wilson RF (eds) Peanuts: Genetics, processing, and utilization. Academic Press, Cambridge, pp 27-66
Tallury SP, Isleib TG, Copeland SC, Rosas-Anderson P, Balota M, Singh D, Stalker HT (2014) Registration of two multiple disease-resistant peanut germplasm lines derived from Arachis cardenasii Krapov.\&nbsp;\& W.C. Gregory, GKP 10017. J Plant Regist 8(1):86-89

Zhan Z, Nwafor CC, Hou Z, Gong J, Zhu B, Jiang Y, Zhou Y, Wu J, Piao Z, Tong Y, Liu C, Zhang C (2017) Cytological and morphological analysis of hybrids between Brassicoraphanus, and Brassica napus for introgression of clubroot resistant trait into Brassica napus L. PLoS ONE 12(5):e0177470. https://doi.org/10.1371/journal.pone. 0177470

Publisher's Note Springer Nature remains neutral with regard to jurisdictional claims in published maps and institutional affiliations. 